\title{
Physical Activity in Patients with Metabolic Syndrome: At Screening and Three Years Thereafter
}

\author{
Hanneke Jansen, MD, PhD, Corine den Engelsen, MD, and Guy E.H.M. Rutten, MD, PhD
}

\begin{abstract}
Background: Metabolic syndrome is a cluster of risk factors for cardiovascular disease and type 2 diabetes. Physical activity can decrease these risks. Many randomized clinical trials to increase physical activity have demonstrated disappointing results, and implementation in daily practice appeared to be difficult. The aim of this study was to investigate whether 3 years of usual care with available guidelines in a primary care setting result in change in physical activity in patients with screen-detected metabolic syndrome.

Methods: After a population-based screening, 473 patients were diagnosed with metabolic syndrome and received advice to increase physical activity. Three years later, they were invited for follow-up. Physical activity was measured by means of the validated SQUASH questionnaire. The primary outcome measure was: \% of metabolic syndrome patients that fulfill the Dutch Physical Activity Guideline (DPAG) criterion (30 min of moderately intensive physical activity at least 5 days per week) at screening and follow-up.

Results: In the final study population $(n=168)$, the proportion of patients fulfilling the DPAG criterion did not significantly increase between screening $(56.0 \%)$ and follow-up $(60.7 \%)(P=0.29)$. Female gender [odds ratio $(\mathrm{OR})=3.59 ; 95 \%$ confidence interval $(\mathrm{CI}) 1.24-10.39]$ and body mass index (BMI) at baseline (OR=0.82; 95\% CI 0.69-0.97) appeared to be independent predictors of increase in physical activity.

Conclusions: In this real-world setting, despite the advice to increase physical activity, the number of metabolic syndrome patients with sufficient physical activity did not significantly increase after 3 years. This finding confirms the need for an intensified approach to achieve an increase in physical activity in this group, with special attention to men and patients with higher BMI values.
\end{abstract}

\section{Introduction}

$\mathrm{T}$ HE METABOLIC SYNDROME IS a cluster of risk factors for cardiovascular disease and diabetes. Despite the ongoing discussion about its exact definition and clinical usefulness, ${ }^{1}$ it is clear that cardiovascular risk factors tend to cluster and that this combination of risk factors is associated with an approximately two-fold increased risk of incident cardiovascular morbidity and mortality. ${ }^{2}$ The underlying pathophysiology of the metabolic syndrome is thought to be related to insulin resistance often caused by (abdominal) obesity and physical inactivity. Consequently, first-line therapy for the metabolic syndrome is aimed at reducing these underlying causes. Weight reduction and increasing physical activity can reduce all of the risk factors and thereby decrease cardiovascular risk. ${ }^{3,4}$ According to existing guidelines of the Dutch College of General Practitioners, general practitioners (GPs) are advised to screen patients for their cardiovascular risk, and every patient with cardiovas- cular risk factors should be stimulated to increase physical activity to at least the Dutch Physical Activity Guideline (DPAG) criterion (30 min or more of moderately intensive physical activity on at least 5 days per week). ${ }^{5}$ However, the effectiveness of lifestyle advice in the real-world setting is unknown. Many randomized clinical trials to increase physical activity have demonstrated disappointing results, and implementation in daily practice appears to be difficult. ${ }^{6,7}$ The aim of the current study was to investigate the proportion of patients that fulfill the DPAG criterion in a population of 473 patients with screen-detected metabolic syndrome at screening and 3 years thereafter, after a period of usual care in general practice.

\section{Methods}

The study "IJsselstein Screening for Central Obesity to Detect Metabolic Syndrome" was conducted in the city of IJsselstein (The Netherlands) among 12,000 individuals., 
The study was approved by the medical ethics committee of the University Medical Centre Utrecht, The Netherlands. Participants, aged 20-70 years and not known to have diabetes, hypertension, dyslipidemia, or cardiovascular disease at the time of screening, were invited to measure their waist circumference at home with a mailed tape measure. People with an increased self-measured waist circumference ( $\geq 102 \mathrm{~cm}$ for men, $\geq 88 \mathrm{~cm}$ in women) were invited for further examinations. A total of 1721 individuals had an increased self-measured waist circumference and underwent a physical examination and laboratory assessment. They were advised to contact their general practice for the results. Metabolic syndrome was defined according to the redefined National Cholesterol Education Program Adult Treatment Panel III (NCEP ATP III) criteria. ${ }^{10}$

Of the 1721 participants, 473 (27\%) fulfilled these metabolic syndrome criteria and 1248 did not. All 473 patients with newly diagnosed metabolic syndrome were invited to visit the practice nurse. The guideline "Cardiovascular Risk Management" of the Dutch College of General Practitioners was available for the practice nurse. ${ }^{5}$ The consequences of the presence of metabolic syndrome were explained to participants, and they received lifestyle advice. We assume that if a cardiovascular risk profile was recorded in the electronic medical file, the patient was indeed educated about his or her health status and received lifestyle advice, including the advice to increase level of physical activity to at least the DPAG criterion.

Three years after screening, 406 metabolic syndrome patients were invited for follow-up measurement; 67 patients were not eligible for follow-up measurements because they had moved, were critically ill, or for other reasons. Of these 406 patients, 194 patients participated in follow-up measurements, and for 188 of them data about physical activity were available both at baseline and follow-up. From data in the electronic medical records, we were able to assess what happened in the first year after screening, including recording of a cardiovascular risk profile. We excluded patients in whom no risk profile was recorded $(n=20)$, because it is unclear whether or not they received the advice to increase their level of physical activity. Consequently, the final study population consisted of 168 patients. Written informed consent was obtained from all participants.

\section{Measurements}

The same measurements were performed both at screening and at follow-up. Body weight, height, waist circumference, and blood pressure were measured. ${ }^{8}$ Venous blood samples were drawn after an overnight fast to determine fasting blood glucose, high-density lipoprotein cholesterol (HDL-C), and triglycerides. Participants completed a questionnaire to determine ethnicity, education level, and physical activity. Ethnicity was defined as Western ethnicity or non-Western ethnicity. Level of education was dichotomized; high was defined as having completed a level of secondary education that permits entry to college. Physical activity was assessed by the SQUASH questionnaire. ${ }^{11}$ This is a validated questionnaire to order subjects according to their level of physical activity and to assess compliance to physical activity guidelines. It contains questions about multiple activities referring to a normal week in the past months. The DPAG, in line with international guidelines, states that every adult should accumulate $30 \mathrm{~min}$ or more of moderately intensive physical activity [ $\geq 4$ metabolic equivalents (MET)] on at least 5 days per week. ${ }^{12-14}$ With the SQUASH questionnaire, we assessed whether patients fulfilled this DPAG criterion at screening and at follow-up. We defined four groups of change in the category of physical activity: Group A, no change, people in this group fulfilled the DPAG criterion both at baseline and at follow-up; group $\mathrm{B}$, no change, people in this group did not fulfill the DPAG criterion both at baseline and at follow-up; group C, decrease, people in this group fulfilled the DPAG criterion at screening but not at follow-up; group D, increase, people in this group did not fulfill the DPAG criterion at screening but did at follow-up.

\section{Outcome measurements}

The primary outcome measurement was the proportion of patients with and without metabolic syndrome that fulfilled the DPAG criterion at screening and at follow-up. The secondary outcome measurements were: (1) Proportion of metabolic syndrome patients that increased and decreased physical activity; (2) predictors of increase and decrease in physical activity; and (3) change in clinical measures between screening and follow-up.

\section{Statistical analysis}

Baseline characteristics were compared between patients with complete data on physical activity both at baseline and follow-up who were advised to increase physical activity (group I) and patients without complete data on physical activity at baseline and follow-up or were not advised to increase physical activity (group II) with a Student $t$-test (for continuous measures) and the Fisher exact test (for dichotomous measures). Percentages of patients with and without metabolic syndrome fulfilling the DPAG criterion at baseline were compared using the Fisher exact test; percentages of metabolic syndrome patients fulfilling the DPAG criterion at baseline and follow-up were compared using a McNemar test. Predictors of increase and decrease in physical activity were investigated by logistic regression analysis.

Predictors of increase were only investigated in patients who did not already fulfill the DPAG criterion at baseline (groups B + D). Predictors of decrease were only investigated in patients who had already fulfilled the DPAG criterion at baseline (groups $\mathrm{A}+\mathrm{C}$ ). Gender-specific z-scores were calculated for waist circumference to take into account differences between sexes and were used in the modeling. First, all potential predictors were tested in a univariate logistic regression analysis. Subsequently, all variables that were associated with increase/decrease in physical activity at the level of $P<0.10$ were entered into a forward-stepwise (likelihood ratio) binary logistic regression analysis to find independent predictors of increase/decrease in physical activity. Because weight and BMI were highly correlated, we only used BMI in the modeling. To compare clinical measures between baseline and follow-up, we used paired-samples $t$-tests. A $P$ value $<0.05$ was considered significant. Analyses were performed using SPSS version 17.0 [SPSS (IBM), Chicago, IL].

\section{Results}

Baseline characteristics of the study population are described in Table 1. Patients in group I, with complete data on 
Table 1. Baseline Characteristics of the Study Population

\begin{tabular}{|c|c|c|c|c|c|c|}
\hline & \multicolumn{2}{|c|}{$\begin{array}{c}\text { All patients with screen- } \\
\text { detected metabolic syndrome }(\mathrm{n}=473)\end{array}$} & \multicolumn{2}{|c|}{ Group $I(\mathrm{n}=168)$} & \multicolumn{2}{|c|}{ Group I $(\mathrm{n}=305)$} \\
\hline & Mean (SD) & $\mathrm{n}(\%)$ & Mean (SD) & $\mathrm{n}(\%)$ & Mean (SD) & $\mathrm{n}(\%)$ \\
\hline Age (years) & $49.1(10.4)$ & & $50.0(9.9)$ & & $48.6(10.7)$ & \\
\hline Gender: male (\%) & & $234(49.5)$ & & $88(52.4)$ & & $146(47.9)$ \\
\hline Western ethnicity (\%) & & $443(93.7)$ & & $163(97.0)^{*}$ & & $280(91.8)$ \\
\hline Higher educational level (\%) & & $137(29.3)$ & & $58(34.5)$ & & 79 (26.3) \\
\hline Weight (kg) & $93.3(15.2)$ & & $92.4(14.7)$ & & $93.8(15.5)$ & \\
\hline $\operatorname{BMI}\left(\mathrm{kg} / \mathrm{m}^{2}\right)$ & $30.5(3.9)$ & & $30.0(3.5)^{*}$ & & $30.7(4.1)$ & \\
\hline Waist circumference men $(\mathrm{cm})$ & $101.1(7.2)$ & & $109.6(7.0)$ & & $110.4(7.3)$ & \\
\hline Waist circumference women $(\mathrm{cm})$ & $100.8(9.8)$ & & $99.1(8.4)^{*}$ & & $101.7(10.3)$ & \\
\hline Systolic blood pressure (mmHg) & $144.7(15.8)$ & & $143.9(15.0)$ & & $145.1(16.2)$ & \\
\hline Diastolic blood pressure (mmHg) & $88.6(7.9)$ & & $88.2(7.4)$ & & $88.8(8.2)$ & \\
\hline Fasting blood glucose (mmol/L) & $5.4(1.3)$ & & $5.3(1.2)$ & & $5.4(1.4)$ & \\
\hline HDL-C men (mmol/L) & $1.1(0.3)$ & & $1.1(0.3)$ & & $1.1(0.2)$ & \\
\hline HDL-C women (mmol/L) & $1.3(0.3)$ & & $1.3(0.3)$ & & $1.3(0.3)$ & \\
\hline Triglycerides $(\mathrm{mmol} / \mathrm{L})$ & $2.1(1.1)$ & & $2.2(1.2)$ & & $2.1(1.0)$ & \\
\hline DPAG criterion at baseline: Yes (\%) & & $254(55.2)$ & & $94(56.0)$ & & $160(54.8)$ \\
\hline
\end{tabular}

Group I: Patients with complete data on physical activity both at baseline and follow-up and who were advised to increase physical activity $(n=168)$.

Group II: Patients without complete data on physical activity at baseline and follow-up or who were not advised to increase physical activity $(n=305)$.

${ }^{*} p<0.05$ : Group I versus Group II.

SD, standard deviation; BMI, body mass index; HDL-C, high-density lipoprotein cholesterol; DPAG, Dutch Physical Activity Guideline.

physical activity at baseline and follow-up and who were advised on physical activity, had a significantly lower body mass index (BMI) and a lower waist circumference (only significant in women) compared to patients without complete data on physical activity at baseline and follow-up or who were not advised on physical activity (group II). In addition, the percentage of patients with Western ethnicity was significantly higher in group I. The percentage of patients that fulfilled the DPAG criterion at baseline did not differ between groups I and II.

\section{DPAG criterion at screening and follow-up}

At screening, $55.2 \%$ of the 460 metabolic syndrome patients who completed the questionnaire fulfilled the DPAG criterion compared to $61.2 \%$ of the 1218 patients without metabolic syndrome $(P=0.03)$. In the group of 168 metabolic syndrome patients who completed questionnaires both at baseline and at follow-up, the proportion of patients fulfilling the DPAG criterion did increase between screening and follow-up, although not significantly: At screening $56.0 \%$ versus $60.7 \%$ at follow-up $(P=0.29)$. In these 168 metabolic syndrome patients, $15.5 \%$ changed from not fulfilling the DPAG criterion at screening to fulfilling the DPAG criterion at follow-up (group D) and 10.7\% changed from fulfilling the DPAG criterion at screening to not fulfilling the DPAG criterion at follow-up (Group C).

In the group of 74 metabolic syndrome patients who did not fulfill the DPAG criterion at screening (groups B+D), 26 $(35.1 \%)$ patients increased physical activity to a sufficient level and fulfilled the DPAG criterion at follow-up. In these 74 metabolic syndrome patients, age, waist circumference gender-specific $z$-score, educational level, and ethnicity were not associated with an increase in physical activity. Univariate logistic regression analyses showed that female gen- der [odds ratio $(\mathrm{OR})=3.67 ; 95 \%$ confidence interval $(\mathrm{CI})$ 1.34-10.03; $P=0.01]$, weight $(\mathrm{OR}=0.96 ; 95 \%$ CI $0.93-0.99$; $P=0.02)$, and BMI (OR=0.80; 95\% CI 0.67-0.96; $P=0.02)$ were associated with an increase in physical activity. Multiple logistic regression analysis revealed that female gender $(\mathrm{OR}=3.59$; 95\% CI 1.24-10.39; $P=0.02)$ and BMI $(\mathrm{OR}=0.82$; 95\% CI 0.69-0.97; $P=0.02$ ) were independent predictors of an increase in physical activity between screening and follow-up.

In the group of 94 metabolic syndrome patients who did fulfill the DPAG criterion at screening (group A+C), 18 $(19.1 \%)$ patients decreased their level of physical activity and did not fulfill the DPAG criterion at follow-up anymore. Age, gender, weight, BMI, waist circumference gender-specific z-score, educational level, and ethnicity were all not significantly associated with a decrease in physical activity.

\section{Changes in clinical measures and metabolic syndrome status}

Table 2 displays clinical measures at screening and followup and the change in these clinical measures between screening and follow-up. Mean weight, BMI, and all metabolic syndrome components except for glucose level improved in all metabolic syndrome patients, regardless of their change in physical activity. But these changes in clinical measures are not significant in all the different categories of change in physical activity. In the total study population of 168 patients, $76(45.2 \%)$ patients still met the criteria for metabolic syndrome at follow-up; this proportion is lowest in patients who decreased their level of physical activity $(n=7,38.9 \%)$.

\section{Discussion}

Three years after being diagnosed with metabolic syndrome and receiving advice to increase the level of physical 
Table 2. Change in Clinical Measures Between Screening and Follow-Up in Patients with Screen-Detected Metabolic Syndrome

\begin{tabular}{|c|c|c|c|c|c|c|c|c|c|c|}
\hline & \multicolumn{2}{|c|}{$A l l^{\mathrm{a}}: \mathrm{n}=168$} & \multicolumn{2}{|c|}{$\begin{array}{c}\text { Group } A \\
\text { No change: }+/+^{\mathrm{b}} \\
\mathrm{n}=76\end{array}$} & \multicolumn{2}{|c|}{$\begin{array}{c}\text { Group B } \\
\text { No change: }-/-^{\mathrm{b}} \\
\mathrm{n}=48\end{array}$} & \multicolumn{2}{|c|}{$\begin{array}{c}\text { Group C } \\
\text { Decrease: }+/{ }^{\mathrm{b}} \\
\mathrm{n}=18\end{array}$} & \multicolumn{2}{|c|}{$\begin{array}{c}\text { Group D } \\
\text { Increase: }-/+^{\mathrm{b}} \\
\mathrm{n}=26\end{array}$} \\
\hline & Mean & $S D$ & Mean & $S D$ & Mean & $S D$ & Mean & $S D$ & Mean & $S D$ \\
\hline Weight at Screening & 92.4 & 14.7 & 89.9 & 14.3 & 98.4 & 12.9 & 90.5 & 14.4 & 90.0 & 16.8 \\
\hline Change in weight $(\mathrm{kg})$ & $-2.7 t$ & 5.9 & $-3.7+$ & 5.8 & -1.7 & 6.3 & -2.1 & 5.2 & -2.0 & 5.9 \\
\hline BMI at screening $\left(\mathrm{kg} / \mathrm{m}^{2}\right)$ & $30.0^{\circ}$ & 3.5 & 30.2 & 3.6 & 30.7 & 3.6 & 29.1 & 2.4 & 28.5 & 3.1 \\
\hline Change in BMI $\left(\mathrm{kg} / \mathrm{m}^{2}\right)$ & $-1.0 \%$ & 1.9 & $-1.4 \dagger$ & 2.0 & $-0.7 \dagger$ & 1.9 & -0.8 & 1.6 & -0.6 & 2.1 \\
\hline Waist circumference at screening $(\mathrm{cm})$ & 104.6 & 9.3 & 103.8 & 9.3 & 108.0 & 8.8 & 102.7 & 9.2 & 101.7 & 8.8 \\
\hline Change in waist circumference $(\mathrm{cm})$ & $-4.0 \%$ & 6.3 & $-5.1 \%$ & 6.3 & $-3.0 \dagger$ & 6.3 & -2.6 & 6.1 & $-3.4 \dagger$ & 6.2 \\
\hline $\begin{array}{l}\text { Systolic blood pressure at } \\
\text { screening }(\mathrm{mmHg})\end{array}$ & 143.9 & 15.0 & 147.6 & 17.3 & 140.4 & 12.2 & 142.5 & 13.6 & 140.6 & 10.7 \\
\hline Change in systolic blood pressure $(\mathrm{mmHg})$ & $-8.9 \div$ & 12.3 & $-9.4 \%$ & 14.0 & $-8.5 t$ & 10.4 & $-5.9 \dagger$ & 11.5 & $-10.3 \ddagger$ & 10.9 \\
\hline FBG at screening $(\mathrm{mmol} / \mathrm{L})$ & 5.3 & 1.2 & 5.2 & 0.7 & 5.3 & 1.1 & 5.0 & 0.6 & 5.5 & 2.3 \\
\hline Change in FBG $(\mathrm{mmol} / \mathrm{L})$ & 0.1 & 0.9 & $0.2 \dagger$ & 0.5 & 0.2 & 0.9 & 0.1 & 0.4 & -0.1 & 1.8 \\
\hline HDL-C at screening $(\mathrm{mmol} / \mathrm{L})$ & 1.2 & 0.3 & 1.3 & 0.3 & 1.1 & 0.2 & 1.2 & 0.3 & 1.3 & 0.5 \\
\hline Change in HDL-C (mmol/L) & $0.1+$ & 0.2 & $0.1 \dagger$ & 0.2 & $0.1+$ & 0.2 & 0.1 & 0.2 & 0.1 & 0.2 \\
\hline Triglycerides at screening $(\mathrm{mmol} / \mathrm{L})$ & 2.2 & 1.2 & 2.1 & 1.0 & 2.4 & 1.1 & 1.8 & 0.6 & 2.4 & 1.8 \\
\hline Change in triglycerides $(\mathrm{mmol} / \mathrm{L})$ & $\begin{array}{c}-0.3 \dagger \\
n\end{array}$ & $\begin{array}{l}1.1 \\
(\%)\end{array}$ & $\begin{array}{c}-0.3 \dagger \\
n\end{array}$ & $\begin{array}{l}1.0 \\
(\%)\end{array}$ & $\begin{array}{c}-0.4 \uparrow \\
n\end{array}$ & $\begin{array}{r}0.9 \\
(\%)\end{array}$ & $\begin{array}{l}0.0 \\
n\end{array}$ & $\begin{array}{r}0.7 \\
(\%)\end{array}$ & $\begin{array}{c}-0.5 \\
n\end{array}$ & $\begin{array}{r}1.7 \\
(\%)\end{array}$ \\
\hline $\begin{array}{l}\text { Number of patients with metabolic } \\
\text { syndrome at follow-up at follow-up }\end{array}$ & 76 & 45.2 & 37 & 48.7 & 20 & 41.7 & 7 & 38.9 & 12 & 46.2 \\
\hline
\end{tabular}

${ }^{a}$ All metabolic syndrome patients with complete data on physical activity at baseline and follow-up after advice to increase level of physical activity; groups A-D.

${ }^{\mathrm{b}}+=$ fulfil the DPAG criterion, at screening/at follow-up; - = not fulfil the DPAG criterion, at screening/at follow-up.

${ }^{\dagger} p<0.05$; paired-samples $t$-test, at screening versus at follow-up.

${ }^{t} p<0.001$; paired-samples $t$-test, at screening versus at follow-up.

SD, standard deviation; BMI, body mass index; FBG, fasting blood glucose; HDL-C, high-density lipoprotein cholesterol; MetS, metabolic syndrome.

activity, the proportion of metabolic syndrome patients fulfilling the DPAG criterion increased, but not significantly. Approximately one-third of the people that did not fulfill the DPAG criterion at baseline achieved the target level of physical activity at follow-up, but especially men and patients with higher BMI seemed to have difficulties in meeting the DPAG criterion. However, mean weight, BMI, and all metabolic syndrome components except for glucose level improved in all metabolic syndrome patients, regardless of their change in physical activity.

According to existing guidelines, overweight patients with one or more cardiovascular risk factors should be stimulated by their GP or practice nurse to become more physically active. ${ }^{5}$ Several lifestyle interventions have been developed to get these patients more physically active. A recent systematic review of 13 trials determined whether or not trials to promote physical activity, based in primary care, showed sustained effects on physical activity in sedentary adults. ${ }^{15}$ They found a small-to-medium improvement in selfreported physical activity at 12 months $(\mathrm{OR}=1.42 ; 95 \% \mathrm{CI}$ 1.17-1.73). Most interventions took place in primary care, included health professionals in delivery, and involved advice or counseling given face to face or by phone on multiple occasions. All studies included only sedentary adults, selfreported measures of physical activity were used, and follow-up was limited to 12 months. Although all interventions are potentially reproducible in real-life settings, most involved multiple contacts over time. Also in patients with impaired glucose tolerance, positive effects of combined lifestyle interventions have been shown, ${ }^{16}$ but the implementation of these interventions in daily practice remains challenging. Kujala et al. investigated the association between increase in physical activity and changes in cardiometabolic risk factors during a lifestyle intervention program in a routine clinical setting. Of the 1871 nondiabetic participants, only $16.6 \%$ reported to have increased their level of physical activity during the previous year. ${ }^{6}$ Also the effects of a Dutch nationwide lifestyle program, with the aim of increasing physical activity and improving dietary behavior in primary care patients with prediabetes or type 2 diabetes, were small and not statistically significant or clinically relevant. $^{7}$

To succeed in increasing physical activity, a change is needed in the practice of GPs as well as in the beliefs and attitudes of the patients. In the EUROPREVIEW survey in the general population, a discrepancy was found between the expectations of patients and the performance of GPs. In addition, $48 \%$ of the 7947 participants aged 30-70 years thought they had to improve their level of physical activity. More than half of the patients said they had plans to change and two-thirds said they were confident of succeeding. This underlines our findings that there is still a high proportion of primary care patients with an unhealthy lifestyle that do not feel the need to change this. However, also only about half of the patients reported that GPs had addressed these topics. ${ }^{17}$ Although this survey included patients from the general population, thus not a selected population of patients diagnosed with metabolic syndrome as in our study, these 
findings reveal the difficulties GPs are facing in carrying out health promotion activities. Although GPs believe they should advise preventive and health promotion activities, in practice they are less likely to do so. Important barriers in prescribing physical activity were lack of time, no reimbursement, lack of knowledge and training, and patients' noncompliance. ${ }^{18-20}$

To conclude, patients do not acknowledge the problem of being physically inactive, and GPs have difficulties encountering the problem of physical inactivity in their patients. Against this background, the results of our study may be regarded both positive and disappointing. Only one-third of the people that did not fulfill the DPAG criterion at baseline achieved the target level of physical activity at follow-up; however, over a 3-year period, mean BMI and all metabolic syndrome components except for glucose level improved in all metabolic syndrome patients.

This discrepancy probably results from the way level of physical activity and especially the change in level of physical activity was measured. As stated by the developers, the SQUASH questionnaire is a fairly reliable and reasonably valid questionnaire to order subjects according to their level of physical activity. Consequently, it is not a continuous measure of physical activity, which would have been much better to measure the change in level of physical activity. Especially in patients with a sedentary lifestyle, a small increase in physical activity can have a beneficial effect on weight and other cardiovascular risk factors. $^{21}$

Unfortunately, we were not able to properly measure small increases in level of physical activity. In general, appropriately measuring (change in) physical activity has been a challenge for many years. ${ }^{22}$ A second limitation of our study regards the study population. Unfortunately, at follow-up we could only obtain complete data in 168 of the 473 patients who were detected with metabolic syndrome at baseline. Patients with a lower BMI and Western ethnicity were more willing to participate in the follow-up measurements. Because patients with a higher BMI are less likely to increase physical activity, the number of patients who fulfilled the DPAG criterion at follow-up and/or increased physical activity might have been even lower in the general population.

In conclusion, only $55.2 \%$ of the metabolic syndrome patients have a sufficient level of physical activity at baseline, and this percentage is not significantly increased after 3 years of usual care in a primary care setting. Given the high risk for future cardiovascular disease, this study confirms the need for a different approach to achieve an increase in physical activity in these patients, with special attention to men and patients with a higher BMI.

\section{Acknowledgments}

The authors express their special thanks to the general practitioners, nurse practitioners, and assistants from general practices Ametisthof, Glennhof, De Poort, 't Steyn, and De Weegbree in IJsselstein, The Netherlands. This work was supported in part by a research grant from the Investigator Initiated Studies Program of Merck Sharp \& Dohme Corp. The opinions expressed in this paper are those of the authors and do not necessarily represent those of Merck Sharp \& Dohme Corp.

\section{Author Disclosure Statement}

No competing financial interests exist.

\section{References}

1. Kahn R. Metabolic syndrome-what is the clinical usefulness? Lancet 2008;371:1892-1893.

2. Dekker JM, Girman C, Rhodes T, et al. Metabolic syndrome and 10-year cardiovascular disease risk in the Hoorn Study. Circulation 2005;112:666-673.

3. Dagogo-Jack S, Egbuonu N, Edeoga C. Principles and practice of nonpharmacological interventions to reduce cardiometabolic risk. Med. Princ Pract 2010;19:167-175.

4. Mujica V, Urzua A, Leiva E, et al. Intervention with education and exercise reverses the metabolic syndrome in adults. J Am Soc Hypertens 2010;4:148-153.

5. The NHG-Guideline Cardiovascular risk managment (first update). Huisarts Wet 2012;55:14-28.

6. Kujala UM, Jokelainen J, Oksa H, et al. Increase in physical activity and cardiometabolic risk profile change during lifestyle intervention in primary healthcare: 1-year follow-up study among individuals at high risk for type 2 diabetes. BMJ Open 2011;1:e000292.

7. Linmans JJ, Spigt MG, Deneer L, et al. Effect of lifestyle intervention for people with diabetes or prediabetes in realworld primary care: Propensity score analysis. BMC Fam Pract 2011;12:95.

8. van den Donk M, Bobbink IW, Gorter KJ, et al. Identifying people with metabolic syndrome in primary care by screening with a mailed tape measure: A survey of 14,000 people in the Netherlands. Prev Med 2009;48:345-350.

9. den Engelsen C, van den Donk M, Gorter KJ, et al. Detection of metabolic syndrome by self-measurement of waist circumference. Ned Tijdschr Geneeskd 2010;154:A1028.

10. Expert Panel on Detection, Evaluation, and Treatment of High Blood Cholesterol in Adults. Executive Summary of The Third Report of The National Cholesterol Education Program (NCEP) Expert Panel on Detection, Evaluation, And Treatment of High Blood Cholesterol In Adults (Adult Treatment Panel III). JAMA 2001;285:2486-2497.

11. Wendel-Vos GC, Schuit AJ, Saris WH, et al. Reproducibility and relative validity of the short questionnaire to assess health-enhancing physical activity. J Clin Epidemiol 2003;56: 1163-1169.

12. Kemper HCG, Ooijendijk WTM, Stiggelbout M. [Consensus about the Dutch Physical Activity Guideline] In Dutch. TSG 2000;78:180-183.

13. Ainsworth BE, Haskell WL, Whitt MC, et al. Compendium of physical activities: an update of activity codes and MET intensities. Med Sci Sports Exerc 2000;32:S498-S504.

14. Pate RR, Pratt M, Blair SN, et al. Physical activity and public health. A recommendation from the Centers for Disease Control and Prevention and the American College of Sports Medicine. JAMA 1995;273:402-407.

15. Orrow G, Kinmonth AL, Sanderson S, et al. Effectiveness of physical activity promotion based in primary care: Systematic review and meta-analysis of randomised controlled trials. BMJ 2012;344:e1389.

16. Tuomilehto J, Lindstrom J, Eriksson JG, et al. Prevention of type 2 diabetes mellitus by changes in lifestyle among subjects with impaired glucose tolerance. N Engl J Med 2001;344: 1343-1350.

17. Brotons C, Drenthen AJ, Durrer D, et al. Beliefs and attitudes to lifestyle, nutrition and physical activity: The views of patients in Europe. Fam Pract 2012;29(Suppl 1):i49-i55. 
18. Attalin V, Romain AJ, Avignon A. Physical-activity prescription for obesity management in primary care: Attitudes and practices of GPs in a southern French city. Diabetes Metab 2012; [Epub ahead of print].

19. Bock C, Diehm C, Schneider S. Physical activity promotion in primary health care: Results from a German physician survey. Eur J Gen Pract 2012;18:86-91.

20. Brotons C, Bjorkelund C, Bulc M, et al. Prevention and health promotion in clinical practice: The views of general practitioners in Europe. Prev Med 2005;40:595-601.

21. Powell KE, Paluch AE, Blair SN. Physical activity for health: What kind? How much? How intense? On top of what? Annu Rev Public Health 2011;32:349-365.
22. Livingstone MB, Robson PJ, Wallace JM, et al. How active are we? Levels of routine physical activity in children and adults. Proc Nutr Soc 2003;62:681-701.

Address correspondence to: Hanneke Jansen, $M D, P h D$

Julius Centre for Health Sciences and Primary Care University Medical Centre Utrecht

P.O. Box 85.500

NL-3508 GA Utrecht

The Netherlands

E-mail: h.jansen-3@umcutrecht.nl 